\title{
Nephrocalcinosis in preterm babies
}

\author{
A Narendra, M P White, H A Rolton, Z I Alloub, G Wilkinson, J H McColl, J Beattie
}

Department of

Neonatology, The Qeen

Mother's Hospital,

Glasgow G3 8SJ, UK

A Narendra

Department of

Paediatrics and

Neonatology, Southern

General Hospital,

Glasgow G51 4TF, UK

M P White

Z I Alloub

Department of

Biochemistry, Stobhill

Hospital, North

Glasgow University

Hospitals NHS Trust,

Balomock Road,

Glasgow G21 3UW, UK

H A Rolton

Department of

Radiology, The Royal

Hospital for Sick

Children, Yorkhill,

Glasgow G3 8SJ, UK

$\mathrm{G}$ Wilkinson

Department of

Statistics, University of

Glasgow, Glasgow

G12 8QQ, UK

$\mathrm{J}$ H McColl

The Renal Unit, The Royal Hospital for Sick Children, Yorkhill,

Glasgow G3 8SJ, UK

J Beattie

Correspondence to:

Dr Narendra, SCBU,

Homerton Hospital,

Homerton Row, London

E9 6SR, UK

N.Aladangady@qmw.ac.uk

Accepted 14 August 2001

\begin{abstract}
Objectives-To determine prospectively the incidence and cause of nephrocalcinosis in preterm infants.

Study design-Inborn babies of gestation less than 32 weeks or birth weight less than $1500 \mathrm{~g}$ were eligible to be entered into a prospective observational study. Two renal ultrasound scans were performed, the first at 1 month postnatal age and the second at term or discharge. Data were collected on gestation, birth weight, sex, race, family history of renal calculi, oliguria on first day, respiratory support (ventilation, steroid, and oxygen dependency), and use of nephrotoxic drugs (gentamicin, vancomycin, and frusemide). Intake of fluid, calcium, and phosphate and plasma urea, creatinine, calcium, and phosphate were recorded for the first 6 weeks of life. Random urinary calcium/creatinine, oxalate/ creatinine, and urate/creatinine ratios and tubular absorption of phosphate were measured once at term.
\end{abstract}

Results-A total of 101 preterm infants were studied. Twenty three (23\%) had abnormal ultrasound scans. Sixteen (16\%) had nephrocalcinosis. On univariate analysis, gestational age, male sex, duration of ventilation, oxygen dependency, duration and frequency of gentamicin treatment, toxic gentamicin/vancomycin levels, and postnatal dexamethasone were significantly associated with nephrocalcinosis. In addition, babies with nephrocalcinosis had a lower intake of fluid, calcium, and phosphate, longer duration of total parenteral nutrition, and higher urinary oxalate/creatinine and uratel creatinine ratios than infants who did not have the condition. There was also a significant association with plasma urea and creatinine but not with plasma calcium or phosphate or urinary calcium. Multivariate analysis showed that the strongest predictors of nephrocalcinosis were duration of ventilation, toxic gentamicin/vancomycin levels, low fluid intake, and male sex.

Conclusion-16\% of babies born at less than 32 weeks gestation developed nephrocalcinosis. The multifactorial origin, in particular, the association with extreme prematurity and severity of respiratory disease, is confirmed. In addition, an association with male sex, frequency and duration of gentamicin use, and high urinary oxalate and urate excretion is shown. (Arch Dis Child Fetal Neonatal Ed 2001;85:F207-F213)

Keywords: nephrocalcinosis; renal dysfunction; calcium; kidney
Nephrocalcinosis in preterm babies was first described in 1982 by Hufnagle et al, who attributed the cause primarily to frusemide treatment. ${ }^{1}$ Since then studies published in the North American literature have shown a varying incidence of $28 \%{ }^{2}$ to $64 \%^{3}$ in very low birthweight babies. In a previous study in the United Kingdom, ${ }^{4}$ the incidence of nephrocalcinosis was $21.5 \%$ and renal calcification (nephrocalcinosis and renal stones) $27 \%$, and in a recent Scandinavian study ${ }^{5}$ an incidence of $20 \%$ was reported.

The role of frusemide in the development of nephrocalcinosis in preterm babies remains unclear. Jacinto et al ${ }^{\beta}$ studied 31 babies of less than $1500 \mathrm{~g}$ birth weight and found a significant association between administration of frusemide and nephrocalcinosis. In a larger study of 79 babies of less than 32 weeks gestation by Short and Cooke, ${ }^{4}$ there was no difference in the mean total dose of frusemide given before detection of renal calcification between those with nephrocalcinosis and the unaffected group. These authors suggested that frusemide was used for infants already at risk of renal calcification and found duration of oxygen dependency to be the strongest predictor of renal calcification. In contrast, Saarela et $a l^{\bar{p}}$ found that the cumulative dose of frusemide in the first 8 weeks of life was significantly greater in the group that developed calcification.

Karlowicz et al studied 50 babies of less than $1200 \mathrm{~g}$ birth weight and found white race and family history of renal calculi to be significantly associated with nephrocalcinosis but not administration of frusemide. The presence of hypercalciuria has also been variably associated with nephrocalcinosis, being significant in some studies ${ }^{2}{ }^{4}$ but not all. ${ }^{3}$ Most of the reports on nephrocalcinosis in preterm infants were before the routine use of antenatal steroids and postnatal treatment with surfactant, both of which have been shown to improve considerably the outcome of very low birthweight babies.

Nephrocalcinosis appears to resolve spontaneously in $40-50 \%$ of cases on follow up, ${ }^{5-10}$ but can be associated with recurrent urinary tract infections, renal colic, and haematuria. ${ }^{8}{ }^{10}$ More controversial is whether nephrocalcinosis leads to a reduction in glomerular filtration rate and tubular dysfunction ${ }^{11}$ or whether renal dysfunction on follow up reflects prematurity. ${ }^{9}$

Our study was designed to establish prospectively the current incidence of nephrocalcinosis in a group of very preterm infants, to study the causes, and to provide a cohort for follow up.

\section{Patients and methods}

Only inborn babies of less than 32 weeks gestation or with a birth weight of less than 
$1500 \mathrm{~g}$ from Queen Mother's Hospital (QMH) and Southern General Hospital (SGH) Glasgow were recruited. Babies born with major congenital anomalies or those who died or were transferred before the end of the study period were excluded. The study protocol was approved by the local ethics committees, and informed written parental consent was obtained.

Data on the following were recorded: sex, gestation, birth weight, race, family history of renal calculi, oliguria on day 1 , number of days ventilated, number of days in oxygen, oxygen dependency at 36 weeks postconceptional age, antenatal and postnatal use of steroids, administration of surfactant, phosphate supplementation, and treatment with frusemide, aminoglycoside (gentamicin), or vancomycin. High peak and trough levels of vancomycin and gentamicin (above normal laboratory reference) were also recorded. The actual daily intake of fluid, calcium, and phosphate was collected for the first 6 weeks of life.

Plasma concentrations of calcium, phosphate, urea, creatinine, and electrolytes were recorded daily during intensive care treatment and weekly thereafter for the first 6 weeks of life. Random urinary calcium/creatinine, urate/ creatinine, and oxalate/creatinine ratios were measured once at term. Urine specimens were collected into a urine collecting bag or as clean catch specimens. Tubular reabsorption of phosphate was calculated once at term. Plasma electrolytes, including calcium and phosphate, were measured by standard dry chemistry methods with the Ortho Vitrous 750 analyser. Urinary urate, calcium, phosphate, and creatinine were measured with the Vitrous 250 analyser. Urine oxalate was measured enzymatically using the Sigma method modified for use on the Cobas Fara (Sigma UK).

Both neonatal units had a policy of providing oral phosphate supplementation. All enterally fed babies at QMH received $1.5 \mathrm{mmol}$ phosphate $/ \mathrm{kg} /$ day to maintain normal plasma phosphate. Babies from SGH received an appropriate dose of phosphate if plasma phosphate was less than $1.5 \mathrm{mmol} / \mathrm{l}$. Total parenteral nutrition (TPN) solutions in both units provided $1 \mathrm{mmol}$ phosphate/kg/day.

A renal ultrasound scan was performed at 1 month of age and once at term. The ultrasound examination was performed by one consultant paediatric radiologist at $\mathrm{QMH}$ (AGW) and by one consultant radiologist at SGH. When nephrocalcinosis was suspected in babies from $\mathrm{SGH}$, the diagnosis was confirmed by a scan performed by AGW. Both radiologists undertaking the ultrasound examination were unaware of the past and current clinical details of the babies. The ultrasound examination was performed using either an Ultramark 4 or Acuson 128 scanner. With the Ultramark 4, a mechanical sector probe operating at either 10 $\mathrm{MHz}$ or $7.5 \mathrm{MHz}$ was used, and, with the Acuson, either a $7.5 \mathrm{MHz}$ linear array or $7.5 \mathrm{MHz}$ sector probe was used. Nephrocalcinosis was diagnosed according to the criteria of Myracle et al. ${ }^{12}$
STATISTICAL METHODS

The groups of infants with and without nephrocalcinosis were compared using appropriate two sample techniques. Because of the considerable skewness shown by many of the data, the Mann-Whitney U test was performed to compare average levels of the continuous variables. The $\chi^{2}$ test of association was used to analyse binary variables, augmented by Fisher's Exact Test when expected frequencies were too small to justify the $\chi^{2}$ approximation. Individual tests were taken to be significant when $\mathrm{p}<0.05$.

Variables that were significant on the univariate analysis were subsequently entered into a multivariate analysis, namely binary logistic regression, to discover whether a small number of explanatory variables may be particularly important for discrimination between babies in the two groups.

\section{Results}

A total of 160 eligible babies were identified between October 1996 and May 1998. Twenty five died, and 27 were transferred to other hospitals. Five parents refused to give their consent, and two babies did not have the term ultrasound scan. This left 101 babies who completed the study.

\section{ULTRASOUND FINDINGS}

Twenty three babies had an abnormal ultrasound and 16 had nephrocalcinosis. Other abnormalities were hydronephrosis in four and bifid pelvis, ovarian cyst, necrotic papilla, and solitary kidney in one each. Two babies with hydronephrosis had antenatal pelvic dilatation. One baby with a postnatal diagnosis of unilateral hydronephrosis developed nephrocalcinosis in the contralateral kidney. The mean age of diagnosis of nephrocalcinosis was 10.4 weeks or 72.8 days (range $38-132$ days). Nephrocalcinosis was detected in only four $(20 \%)$ babies on the first ultrasound scan performed at 38-46 days of life, and this persisted at term. The nephrocalcinosis was bilateral in 10 and unilateral in six infants; all involved the left kidney. An approximate 95\% confidence interval (CI) for the population rate of nephrocalcinosis was $8.7 \%$ to $23.0 \%$.

BASIC DATA

Lower gestational age and male sex were significantly associated with an increased risk of nephrocalcinosis (table 1). Birth weight, growth retardation, and family history of renal calculi were not significantly associated. There were 10 babies of gestational age $>31-34$ weeks and birth weight below $1500 \mathrm{~g}$ in the study, and none developed nephrocalcinosis. All 16 babies with renal calcification were white but this association was not significant $(p=0.234)$. Only seven babies in the study population were non-white. All babies with nephrocalcinosis were asymptomatic.

VENTILATION AND OXYGEN DEPENDENCY

All the babies with nephrocalcinosis were ventilated compared with 50 (59\%) of those without (table 2). The median duration of ventilation was 7.5 days for babies with 
Table 1 Comparison of the basic data of the infants with or without nephrocalcinosis

\begin{tabular}{llll}
\hline & With $(n=16)$ & Without (85) & p Value \\
\hline Gestation (weeks) & $27(24-31)$ & $30(24-34)$ & 0.0014 \\
Birth weight (g) & $1170(565-1880)$ & $1270(640-2720)$ & $0.099^{\star}$ \\
Male/female ratio & $13 / 3$ & $41 / 44$ & 0.015 \\
SGA/AGA & $2 / 14$ & $24 / 61$ & 0.187 \\
F/H of renal stone (yes/no) & $3 / 13$ & $4 / 81$ & 0.155 \\
\hline
\end{tabular}

Values are median (range).

$\star 95 \%$ Confidence interval 49 to 390 .

SGA, Small for gestational age; AGA, appropriate size for gestational age; F/H, family history

Table 2 Comparison of the respiratory support of the infants with or without nephrocalcinosis

\begin{tabular}{lccc}
\hline & $\begin{array}{l}\text { With } \\
(n=16)\end{array}$ & $\begin{array}{c}\text { Without } \\
(n=85)\end{array}$ & p Value \\
\hline Ventilation & $16(100)$ & $50(59)$ & $<0.001$ \\
Median duration of ventilation (days) & 7.5 & 1 & $<0.0001$ \\
Reventilation, 2 or >2 episodes & $6(37)$ & $7(8)$ & 0.005 \\
Oxygen (median days) & 75.5 & 14 & 0.0013 \\
$\mathrm{O}_{2}$ dependency at 36 wks PCA & $11(69)$ & $25(29)$ & 0.003 \\
\hline
\end{tabular}

Values in parentheses are percentages.

PCA, Postconceptional age.

nephrocalcinosis compared with one day for those without $(95 \%$ CI -13 to -3$)$. Six (37\%) infants with nephrocalcinosis required reventilation twice or more compared with seven (8\%) of those without. Median duration of continuous positive airway pressure was also significantly different between the two groups (95\% CI 0 to 2). The median duration of oxygen dependency for babies with nephrocalcinosis was 75.5 days compared with 14 days for those without ( $95 \%$ CI -79.98 to -7.99 ). Eleven $(69 \%)$ infants with nephrocalcinosis were oxygen dependent at 36 weeks postconceptional age compared with 25 (29\%) of those without.

\section{MEDICATION}

There was no association between antenatal treatment with steroids and nephrocalcinosis (table 3). Nephrocalcinosis, however, was significantly associated with the use of surfactant, postnatal dexamethasone, and frusemide. The median total dose of frusemide given to infants with nephrocalcinosis before detection of calcification on the term ultrasound scan was $16.1 \mathrm{mg}$ (range 5-62), and in infants without nephrocalcinosis it was $24 \mathrm{mg}$ (range 2-195.7), which is not significantly different $(p=0.75)$. None of the four infants in whom nephrocalcinosis was detected on the first scan received frusemide.

Eleven $(69 \%)$ infants with nephrocalcinosis had high trough or peak serum levels of vancomycin or gentamicin compared with the standard laboratory reference range on at least one occasion compared with $17(20 \%)$ of the infants without nephrocalcinosis $(p=0.001)$. This difference remained significant after correction for gestation by logistic regression.

Table 3 Relation between medication and nephrocalcinosis (NC)

\begin{tabular}{lccl}
\hline Drug & $N C(n=16)$ & Non NC $(n=85)$ & p Value \\
\hline Antenatal steroid & $12(75)$ & $69(81)$ & 0.57 \\
Dexamethasone & $6(37)$ & $5(6)$ & 0.001 \\
Surfactant & $15(94)$ & $44(52)$ & 0.002 \\
Frusemide & $9(56)$ & $20(23)$ & 0.008 \\
High nephrotoxic antibiotic level & $11(69)$ & $17(20)$ & 0.001 \\
Phosphate & $13(81)$ & $61(72)$ & 0.432 \\
\hline
\end{tabular}

Values in parentheses are percentages.

${ }^{\star}$ High serum levels of gentamicin and/or vancomycin.
Seventy three infants without nephrocalcinosis and 15 infants with nephrocalcinosis received gentamicin for a median of one and two episodes respectively ( $95 \%$ CI 0.0 to 1.0 ). The median duration of gentamicin treatment was five and 23 days respectively in the two groups (95\% CI 3.0 to 20.0). On further analysis there was a significant association between the number of episodes and duration of gentamicin treatment and nephrocalcinosis $(p=0.0077$ and $\mathrm{p}=0.0002$ respectively). Thirty infants without nephrocalcinosis and 11 with nephrocalcinosis received vancomycin for a median of one (range one to three) and one (range one to three) episodes respectively. The median duration of vancomycin treatment was 9.5 (range 2-70) and 22 (range 1-51) days respectively in the two groups. There was no significant difference in frequency and duration of vancomycin use between the groups $(\mathrm{p}=0.35$ and $\mathrm{p}=0.41$ respectively). The median high gentamicin trough level in the group with nephrocalcinosis was $2.6 \mathrm{mg} / 1$ (range $>2.0-6.0$ ) and $2.8 \mathrm{mg} / 1$ $(>2-4.8)$ in the group without nephrocalcinosis $(\mathrm{p}=0.99)$. No infant had high peak gentamicin level. The median high vancomycin trough was $15 \mathrm{mg} / 1$ (range 13.7-29) in the nephrocalcinosis group and $16.5 \mathrm{mg} / \mathrm{l}$ (range 12.9-22.8) in non-nephrocalcinosis group $(\mathrm{p}=0.88)$. The median high peak serum level of vancomycin was $54.5 \mathrm{mg} / 1$ (range $41.1-100$ ) in the nephrocalcinosis group compared with $43.15 \mathrm{mg} / 1$ (range 41.2-44) in the non-nephrocalcinosis group $(p=0.059)$. Eighty eight babies received gentamicin, and, of these, 41 received vancomycin, 24 concurrently and 17 consecutively. No baby had vancomycin who had not received gentamicin. Receiving vancomycin in addition to gentamicin significantly increased the risk of nephrocalcinosis $(p=0.0225)$, but the risk was no greater when vancomycin was given concurrently compared with consecutively $(p=0.486)$.

\section{NUTRITION}

The median duration of TPN in infants with and without nephrocalcinosis was 18 and seven days respectively $(p=0.0009)$. The median calcium intake was lower in the group with nephrocalcinosis from 2-6 weeks of life, and this difference reached significance during weeks 3, 5, and 6 (fig 1C). Median phosphate intake in the infants with nephrocalcinosis was lower from week 2 , and this difference was significant during weeks 3, 5, and 6 (fig 1B). Babies with nephrocalcinosis had a lower median fluid intake from 2-6 weeks of life, but this was only significant during week 3 (fig 1A).

SERUM BIOCHEMISTRY

Nephrocalcinosis was significantly associated with higher median levels of serum creatinine during weeks 3-5 (fig 2B) and higher median levels of serum urea in weeks 1-5 (fig 2A). There was no significant difference in median levels of plasma calcium or phosphate between babies with nephrocalcinosis and those without (fig 2C,D).

URINARY METABOLITE CONCENTRATIONS

The median urinary oxalate/creatinine and urate/creatinine ratios were significantly higher 

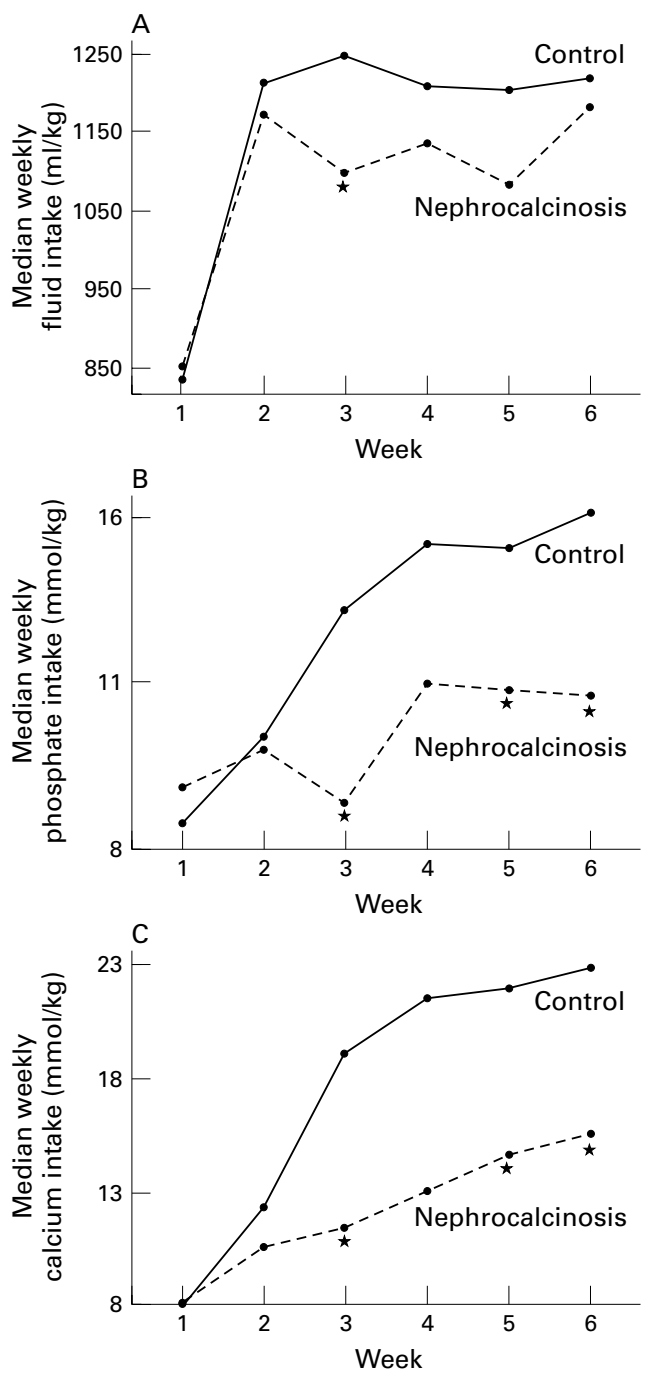

Figure 1 Median weekly fluid (A), phosphate (B), and calcium $(C)$ intake for the first 6 weeks of life in infants with nephrocalcinosis and those without (controls).

*Significantly different from controls.

in babies with nephrocalcinosis (table 4). There was no significant difference in median urinary calcium/creatinine ratio or tubular reabsorption of phosphate between the groups. Only one infant (without nephrocalcinosis) had oliguria on day 1 .

UNIVARIATE ANALYSIS

The most significant of all the above variables was duration of ventilation $(\mathrm{p}=0.0001)$.

\section{MULTIVARIATE ANALYSIS}

This was performed by stepwise fitting of a binary logistic regression test model. The presence/absence of nephrocalcinosis was the dependent variable and the predictor variables were all those that had produced a significant result in the univariate analysis. The strongest indicators of nephrocalcinosis (in order) were: number of days ventilated ( $\mathrm{p}=0.0001)$; toxic gentamicin/vancomycin levels $(p=0.0059)$; fluid intake in week 3 ( $\mathrm{p}=0.0059)$; male sex $(p=0.023)$. These four variables correctly allocated $80.6 \%$ of the sample overall to the nephrocalcinosis or non-nephrocalcinosis group.
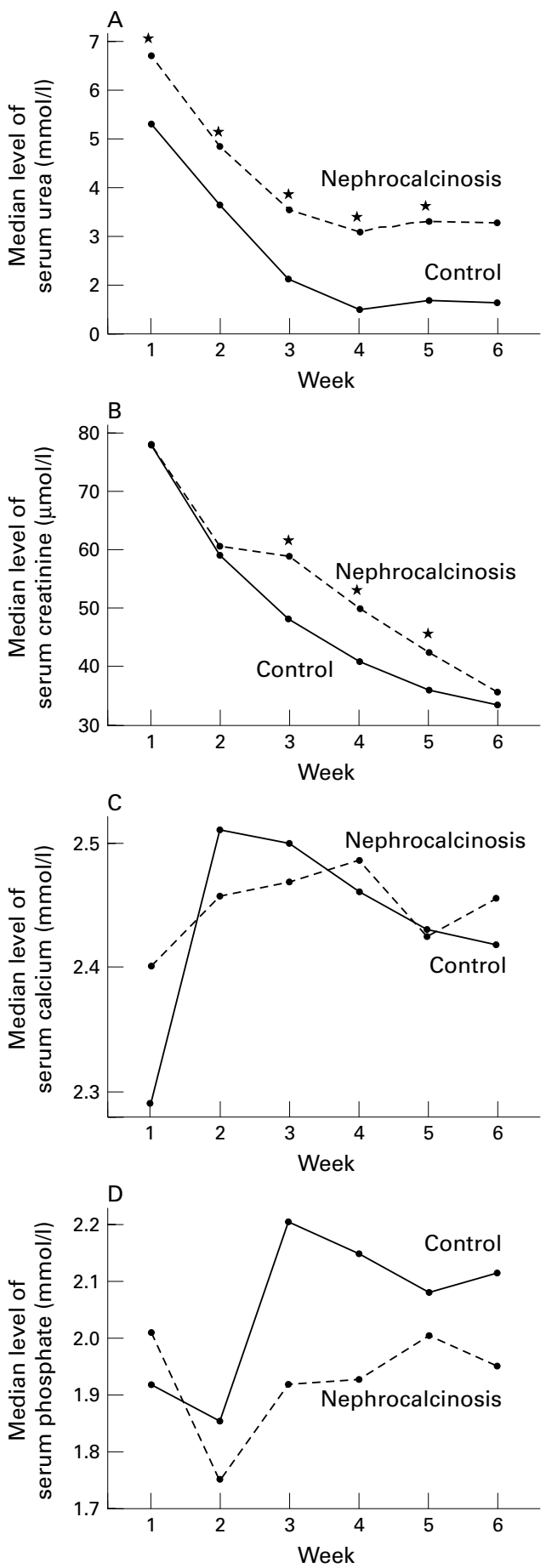

Figure 2 Median serum concentrations of urea $(A)$, creatinine (B), calcium $(C)$, and phosphate $(D)$ for the first 6 weeks of life in infants with nephrocalcinosis and those without (controls). *Significantly different from controls.

\section{Discussion}

The incidence of nephrocalcinosis (16\%) in this study is lower than previously reported, ${ }^{2-5}$ which may reflect improvements in neonatal intensive care, in particular the antenatal use of steroids and surfactant and improved nutrition. Our policy of ensuring adequate oral phosphate supplementation may also have helped to decrease the incidence. However, we note that the wide interval estimate for this 
Table 4 Comparison of urinary metabolite results for infants with or without nephrocalcinosis

\begin{tabular}{|c|c|c|c|}
\hline & With & Without & $p$ Value \\
\hline Oxalate/creatinine ratio & $\begin{array}{l}0.4(0.15-0.89) \\
(\mathrm{n}=13)\end{array}$ & $\begin{array}{l}0.3(0.07-0.95) \\
(\mathrm{n}=56)\end{array}$ & 0.0093 \\
\hline Urate/creatinine ratio & $\begin{array}{l}1.45(0.70-1.94) \\
(\mathrm{n}=13)\end{array}$ & $\begin{array}{l}1.04(0.38-2.39) \\
(\mathrm{n}=51)\end{array}$ & 0.0383 \\
\hline Calcium/creatinine ratio & $\begin{array}{l}1.02(0.11-5.28) \\
(n=16)\end{array}$ & $\begin{array}{l}0.78(0.01-8.32) \\
(\mathrm{n}=72)\end{array}$ & 0.5591 \\
\hline TRP & $\begin{array}{l}86.09(68-100) \% \\
(n=14)\end{array}$ & $\begin{array}{l}83.94(42.7-100) \% \\
(\mathrm{n}=58)\end{array}$ & 0.5350 \\
\hline
\end{tabular}

Values are median (range). $\mathrm{n}=$ Number of babies who had the test.

TRP, Tubular reabsorption of phosphate.

population includes most of the values reported in previous studies and therefore our incidence may not be a true reduction.

We think it unlikely that we underdiagnosed nephrocalcinosis because ultrasound is a very sensitive diagnostic tool. Cramer et $a l^{13}$ reported a sensitivity of $96 \%$ and specificity of $85 \%$ using ultrasound compared with computed tomography and postmortem histological diagnosis in a rabbit model. We used strict criteria $^{12}$ and a single experienced paediatric radiologist to diagnose/confirm the presence of nephrocalcinosis.

In agreement with other studies, we found nephrocalcinosis to be associated with low

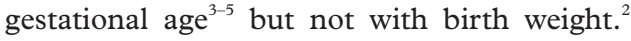
For the first time to our knowledge, we found male sex to be significantly associated with nephrocalcinosis but did not find an association with a family history of renal calculi or white race as reported by Karlowicz et $a l^{2}{ }^{2}$ although the small number of non-white infants $(n=7)$ in this study limited any statistical evaluation.

\section{RESPIRATORY DISEASE}

Short and Cooke ${ }^{4}$ found duration of oxygen to be the strongest clinical indicator of renal calcification. We confirm that severe respiratory disease, as indicated by duration of ventilation, reventilation episodes, duration of oxygen, and oxygen dependence at 36 weeks after conception, is significantly associated. All babies in our study with nephrocalcinosis were ventilated, and duration of ventilation was the most significant variable in the univariate and multivariate analyses. Preterm infants with lung disease are reported to have decreased urinary citrate, which may predispose them to nephrocalcinosis ${ }^{14}$ because citrate is a known inhibitor of renal calcification in adults and children. ${ }^{15} 16$

MEDICATION

We found an association between nephrocalcinosis and postnatal dexamethasone in agreement with the findings of Saarela et $a .^{5}$ Although corticosteroids may cause negative calcium balance, osteopenia, hypercalciuria, and nephrocalcinosis in children, ${ }^{17}$ few data are available on the effect of dexamethasone on urinary calcium excretion in preterm infants. Sonntag and Gaude ${ }^{18}$ found no increase in urinary calcium excretion in a group of preterm infants who received dexamethasone compared with a control group. Postnatal dexamethasone and surfactant may not be independent variables, but merely reflect the severity of the respiratory disease and low gestation.

The role of frusemide in nephrocalcinosis is unclear. Although frusemide produces nephrocalcinosis in a rat model, ${ }^{13}{ }^{19}$ studies on low birthweight babies have shown the use of frusemide to be both significantly ${ }^{1-57810}$ and non-significantly ${ }^{20}$ associated with nephrocalcinosis. The present study shows that the amount of frusemide given before the detection of renal calcification did not differ significantly from that given to unaffected infants. This finding is in agreement with the work of Short and Cooke ${ }^{4}$ but not that of Saarela et al. ${ }^{5}$ This discrepancy may reflect the fact that frusemide is given to babies already at risk of nephrocalcinosis because of other factors such as severe respiratory disease.

We have shown that high nephrotoxic antibiotic levels were strongly associated with nephrocalcinosis independently of gestation. There was no direct relation between the actual toxic levels of gentamicin or vancomycin, but the number of doses and the duration of gentamicin treatment were highly significant. Androniku et $a l^{21}$ reported that therapeutic doses of gentamicin in full term infants resulted in an increase in urinary calcium excretion, which persisted up to 48 hours after discontinuation of treatment. Similar findings of hypercalciuria with therapeutic doses of gentamicin have been shown in a rat model. ${ }^{22}$ Other animal data have shown that the renal toxicity of aminoglycoside is related to frequency and duration of treatment rather than the actual serum toxic level. ${ }^{23}{ }^{24}$ In infants, isolated vancomycin associated nephrotoxicity is rare and unrelated to toxic serum levels, ${ }^{25}$ but a combination with gentamicin can result in nephrotoxicity. ${ }^{26}$ In our study, all babies who received vancomycin also had gentamicin. We were therefore unable to look at the effect of vancomycin alone but showed that the combination of vancomycin and gentamicin significantly increased the risk of nephrocalcinosis. The underlying mechanism is likely to be that of secondary heterogeneous nucleation of calcium phosphate or oxalate crystals in damaged tubular cells. ${ }^{27}$

NUTRITIONAL FACTORS

In this study, TPN was significantly associated with nephrocalcinosis, and we confirm the work of Short and Cooke ${ }^{4}$ showing that nephrocalcinosis is significantly associated with low intake of calcium, phosphate, and fluid and raised serum creatinine and urea. Phosphate deficiency is typically associated with hypercalciuria, whereas phosphate supplementation decreases urinary calcium excretion. ${ }^{28}$ Excess phosphate administration may result in renal rather than skeletal deposition of calcium. ${ }^{29} 30$ However, unlike Saarela et $a l,{ }^{5}$ we did not find phosphate supplementation to be significantly associated with nephrocalcinosis. In our study, 
although we tried to avoid phosphate deficiency, babies with nephrocalcinosis still had a lower phosphate intake, and this was related mainly to the longer duration of TPN. The TPN solution that we used provided $1 \mathrm{mmol}$ phosphate $/ \mathrm{kg} /$ day. There are concerns about the solubility of higher concentrations of phosphate in TPN solutions, although a concentration of $1.34 \mathrm{mmol} / \mathrm{kg} /$ day has been associated with lower urinary calcium excretion. ${ }^{31}$

\section{URINE BIOCHEMISTRY}

We found a significant association between nephrocalcinosis and urinary oxalate/ creatinine and urate/creatinine ratios measured at term. None of our babies were receiving TPN when urinary oxalate and urate were being determined. The role of increased urate and oxalate in the development of renal calcification in children and adults has been well documented, ${ }^{32}{ }^{33}$ but to our knowledge, this has not previously been shown in preterm babies in a prospective study. Calcium oxalate has been shown in a postmortem study to be the most common type of renal calcification in infants dying after intensive care. ${ }^{34}$ The oxalate/ creatinine ratios found in our study are in broad agreement with the high levels quoted in the literature for preterm babies, who are known to have higher oxalate/creatinine ratios than those born at term..$^{35}$ Preterm and healthy term babies have substantially higher oxalate/ creatinine ratios than adults, reaching a peak at 3-4 weeks and declining thereafter ${ }^{36}{ }^{37}$ until 6.5 years of age. ${ }^{38}$ In addition, babies given $\mathrm{TPN}^{39}{ }^{40}$ or formula feeds ${ }^{41}$ have significantly higher oxalate/creatinine ratios. Therefore it is possible that, in the babies in this study, there may have been even higher oxalate excretion during the period of TPN administration before urine samples were collected.

Tsukahara $e t a l^{42}$ has shown that urinary uric acid levels in premature babies remained significantly higher than in normal term infants during the first month of life, and respiratory failure resulted in a further increase. In our study, the urinary urate/creatinine ratios were comparable to the values obtained by Tsukahara in preterm babies with respiratory failure. This may contribute to the high incidence of nephrocalcinosis in babies with lung disease. It can be concluded from this and previous studies that excretion of oxalate and urate is increased in premature infants. Berard et $a l^{43}$ concluded that these two lithogenic factors are at least as important as hypercalciuria, although that study was based on results from a small group of 10 premature babies.

The random urinary calcium/creatinine ratios at term were not significantly associated with nephrocalcinosis, although the values were higher than those quoted by Reusz et al. ${ }^{38}$ We measured urinary calcium once at term, therefore we may have missed significant hypercalciuria during the first 6 weeks of life as reported by Short and Cooke, ${ }^{4}$ or we may have prevented significant calciuria by phosphate supplementation.

Despite the recognised multifactorial origin of nephrocalcinosis, our own data and those of others suggest that certain preventive strategies may be of benefit. Firstly, avoidance of antibiotic toxicity by the use of shorter courses of aminoglycoside and the more frequent use of mathematical modelling is likely to be beneficial. Secondly, phosphate supplementation is also worth considering, and we feel that TPN should ideally provide $1.34 \mathrm{mmol} / \mathrm{kg} / \mathrm{day}$, and, with both intravenous and oral supplementation, the target serum phosphate concentration of $1.5 \mathrm{mmol} / 1$ should be achieved.

In conclusion, we have documented an incidence of nephrocalcinosis of $16 \%$ in infants born at gestation $<32$ weeks. It was particularly associated with severe respiratory disease and only developed in babies who were ventilated. We have shown a new association of nephrocalcinosis with male sex, frequency and duration of gentamicin use, and high urinary excretion of oxalate and urate. The group at risk could be screened at term with one renal ultrasound scan. However, the long term outcome of nephrocalcinosis needs clarification and until then no firm recommendations can be given about screening. Our cohort will be followed up to elucidate further the natural history of nephrocalcinosis. Further studies are also needed on the preventive role of inhibitors of urinary calcification and supplementation with citrate and magnesium.

We are grateful for the contributions of Dr D A R Robertson (renal ultrasound examination) and $\mathrm{Dr} \mathrm{R}$ Logan (urinary biochemistry). We thank the Medical and Nursing staff of the neonatal intensive care units of The Queen Mother's Hospital and Southern General Hospital, Glasgow.

1 Hufnagle KG, Khan SN, Penn D, et al. Renal calcifications: a complication of long-term furosemide therapy in preterm infants. Pediatrics 1982;70:360-3.

2 Karlowicz MG, Katz ME, Adelman RD, et al. Nephrocalcinosis in very low birth weight neonates: family history of kidney stones and ethnicity as independent risk factors. $f$ Pediatr 1993;122:635-8.

3 Jacinto JS, Modanlou HD, Crade M, et al. Renal calcification in very low birth weight infants. Pediatrics

4 Short A, Cooke RWI. The incidence of renal calcification in preterm infants. Arch Dis Child 1991;66:412-17.

5 Saarela T, Vaarala A, Lanning P, et al. Incidence, ultrasonic patterns and resolution of nephrocalcinosis in very low birth weight infants. Acta Paediatr 1999;88:655-60.

6 Modanlou HD, Beharry K, Padilla G, et al. Combined effects of antenatal corticosteroids and surfactant supplementation on the outcome of very low birth weight infants. f Perinatol 1996;16:422-8.

7 Downing GJ, Egelhoff JC, Daily DK, et al. Furosemiderelated renal calcifications in the premature infant: a longitudinal ultrasonographic study. Pediatr Radiol 1991;21:563-5.

8 Ezzedeen F, Adelman RD, Ahlfors CE. Renal calcifications in preterm infants: pathophysiology and long-term sequelea. F Pediatr 1988;113:532-9.

9 Jones CA, King S, Shaw NJ, et al. Renal calcification in preterm infants: follow up at 4-5 years. Arch Dis Child Fetal Neonatal Ed 1997;76: F185-9.

10 Pope JC, Trusler LA, Klein AM, et al. The natural history of nephrocalcinosis in premature infants treated with loop diuretics. F Urol 1996;156:709-12

11 Downing GJ, Egelhoff JC, Daily DK, et al. Kidney function in very low birth weight infants with furosemide-related renal calcifications at ages 1-2 years. $\mathcal{f}$ Pediatr 1992;120:599-604

12 Myracle MR, McGahan JP, Goetzman BW, et al. Ultrasound diagnosis of renal calcification in infants on chronic frusemide therapy. $\mathcal{F}$ Clin Ultrasound $1986 ; 14: 281-7$.

13 Cramer B, Husa L, Pushpanathan C. Nephrocalcinosis in rabbits: correlation of ultrasound, computed tomography, pathology and renal function. Pediatr Radiol 1998;28:9-13.

14 Murphy JL, Mendoza SA. Decreased urinary citrate in preterm infants with lung disease. Child Nephrol Urol 1990;10:76-80.

15 Barcelo P, Wuhl O, Servitge E, et al. Randomised double-blind study of potassium citrate in idiopathic double-blind study of potassium citrate in idiopathic
hypocitrturic calcium nephrolithiasis. 1993;150:1761-4.

16 Ettinger B, Pak CY, Citron JT, et al. Potassium-magnesium citrate is an effective prophylaxis against recurrent calcium oxalate nephrolithiasis. f Urol 1997;158:2069-73. 
17 Stapleton FB, McKay CP, Noe HN. Urolithiasis in children: the role of hypercalciuria. Pediatr Ann 1987;16:980.

18 Sonntag J Gaude M. Effect of dexamethasone and spironolactone therapy in calcium and phosphate homeostasis in preterm infants with a birth weight under $1500 \mathrm{~g}$. Klin Padiatr 1998;210:354-7.

19 Osorio AV, Alon MM, Nichols MA, et al. Effect of age on furosemide-induced nephrocalcinosis in the rat. Biol Neonat 1998;73:306-1

20 Katz ME, Karlowicz G, Adelman R, et al. Nephrocalcinosis in very low birth weight neonates: sonographic patterns, histologic characteristics and clinical risk factors. $f$ Ultrasound Med 1994;13:777-82

21 Andronikou S, Giapros VI, Cholevas VI, et al. Effect of aminoglycoside therapy on renal function in full-term infants. Pediatr Nephrol 1996;10:766-8.

22 Parsons PP, Garland HO, Harpur ES, et al. Acute gentamicin-induced hypercalciuria and hypermagnesiuria in the rat: dose-response relationship and role of renal tubular injury. Br f Pharmacol 1997:122:570-6.

23 Frame PT, Phair JP, Watanakunakorn C, et al. Pharmacologic factors associated with gentamicin nephrotoxicity in logic factors associated with gentam

24 Bennett WM, Plamp CE, Gilbert DN, et al. The influence of dosage regimen on experimental gentamicin nephrotoxicity: dissociation of peak serum levels from renal failure. $\mathcal{f}$ Infect Dis 1979;140:576-80

25 Bhatt-Mehta V, Schumacher RE, Faix RG, et al. Lack of vancomycin-associated nephrotoxicity in newborn infants: a case-control study. Pediatrics 1999;103:e48.

26 Fauconneau B, Favreliere S, Pariat C, et al. Nephrotoxicity of gentamicin and vancomycin given alone and in combination as determined by enzymuria and cortical antibiotic levels in rats. Renal Failure 1997;19:15-22.

27 Adams ND, Rowe JC. Nephrocalcinosis. Clin Perinatol 1992;19:179-95.

28 Holland PC, Wilkinson AR, Diez J, et al. Prenatal deficiency of phosphate, phosphate supplementation, and rickets in of phosphate, phosphate supplementation, and rickets

29 Short A, Shaw NJ, Weindling AM. Nephrocalcinosis and phosphate supplementation in a preterm infant. Acta Paediatr Scand 1990;79:968-9.
30 Matsuzaki H, Uehara M, Suzuki K, et al. High phosphorus diet rapidly induces nephrocalcinosis and proximal tubular diet rapidly induces nephrocalcinosis and proximal tubular
injury in rats. $\mathcal{F}$ Nutr Sci Vitaminol (Tokyo) 1997;43:627injur.

31 Vileisis R. Effect of phosphate intake in TPN infusates in preterm neonates. $\mathscr{F}$ Pediatr 1987;110:586-90.

2 Coe FL. Treated and untreated recurrent calcium nephrolithiasis in patients with idiopathic hypercalciuria, hyperuricosuria or no metabolic disorder. Ann Intern Med 1977;87:404-10.

33 Robertson WG, Peacock $M$. The causes of idiopathic calcium stone disease: hypercalciuria or hyperoxaluria? Nephron 1980;26:105-10.

34 McCormick FCS, Brady K, Keen CE. Oxalate nephrocalcinosis. A study in autopsied infants and neonates. Pediatr Pathol Lab Med 1996;16:479-88.

35 Hoppe B, Hesse A, Neuhaus T, et al. Influence of nutrition on urinary oxalate and calcium in preterm and term on urinary oxalate and calcium in preter
infants. Pediatric Nephrol 1997;11:687-90.

36 Sonntag J, Schaub J. Oxalate excretion during the first 7 weeks in very low birth weight infants. Biol Neonat weeks in very 1 .

37 Leumann EP, Dietl A, Matasovic A. Urinary oxalate and glycolate excretion in healthy infants and children. Pediatr Nephrol 1990;4:493-7.

38 Reusz SS, Dobos M, Byrd D, et al. Urinary calcium and oxalate excretion in children. Pediatr Nephrol 1995;9:39-44.

ppe B, Hesse A, Neuhaus T, et al. Urinary saturation and nephrocalcinosis in preterm infants: effect of parenteral nutrition. Arch Dis Child 1993; 69:299-303.

40 Campfield T, Braden G. Urinary oxalate excetion by very low birth weight infants receiving parenteral nutrition. Pediatrics 1989;84:860-3.

41 Campfield T, Braden G, Flyn-Valone P, et al. Urinary oxalate excetion in premature infants: effect of human milk versus formula feeding. Pediatrics 1994;94:674-8.

42 Tsukahara $\mathrm{H}$, Hiraoka $\mathrm{M}$, Hori $\mathrm{C}$, et al. Urinary uric acid excretion in term and preterm infants. $\mathcal{F}$ Paediatr Child Health 1996;32:330-2

43 Berard E, Dageville C, Bekri S, et al. Nephrocalcinosis and prematurity: importance of urate and oxalate excretion. Nephron 1995;69:237-41.

\section{Archives of Disease in Childhood}

November 2001 issue

The following articles_being published in the November 2001 issue of Archives of Disease in

Childhood - may be of interest to reader of the Fetal and Neonatal edition.

Cerebral palsy: not always what it seems

R Gupta, RE Appleton

Radial osteomyelitis as a complication of venous cannulation

R Straussberg, L Harel, Z Bar-Sever, J Amir

Henna causes life threatening haemolysis in glucose-6-phosphate dehydrogenase deficiency

P Raupp, J Ali Hassan, M Varughese, B Kristiansson 